\title{
Deficits in serum amyloid A contribute to increased neonatal mortality during murine listeriosis
}

\author{
J. Seth Hawkins ${ }^{1}$, QingQing Wu², Yanxia Wang² and Christopher Y. Lu²
}

BACKGROUND: To understand the increased susceptibility of preterm neonates to infection.

METHODS: A murine listeriosis model using immunohistochemistry, microarray technology, and real-time polymerase chain reaction (PCR).

RESULTS: We report that recombinant serum amyloid A (SAA) administered prophylactically $18 \mathrm{~h}$ before intraperitoneal (i.p.) inoculation with Listeria monocytogenes conferred a dramatic survival benefit compared with administration of only vehicle in neonatal mice. Neonates that received the recombinant SAA protein had significantly fewer Listeria colony counts on plating of infected liver and showed significantly more activated macrophages, but SAA did not affect postnatal growth. Real-time $P C R$ was used to confirm the microarray findings that gene expression levels for the SAA proteins 1 (Saa1) and 2 (Saa2), in addition to that for orosomucoid-2 (Orm2), were strikingly elevated in the adult compared with those in the neonate. Realtime PCR analysis showed that of the acute phase cytokines, tumor necrosis factor (TNF) gene expression increased exponentially with time in the infected adult, whereas neonates did not show similar increases.

CONCLUSION: The increased susceptibility of neonatal mice to listeriosis is in part mediated by a deficiency in the acute phase response, specifically expression of SAA, and that prophylactic SAA protein before neonatal murine listeriosis results in more macrophage activation, lower Listeria counts, and greater survival.

A critical component of the response to neonatal infection is mediated by the innate immune system $(1,2)$, which is primarily composed of neutrophils, macrophages, and natural killer cells. Lack of macrophage activation, as determined by expression of major histocompatibility complex (MHC) class-II $(3,4)$ or B7-H1 (5), is a well-known defect in the innate immune response of neonatal mice and is associated with an increased susceptibility to listeriosis. However, macrophage activation is just one component following infection with Listeria monocytogenes. Initially, there is recruitment of macrophages and neutrophils to the site of infection during the first $24-48 \mathrm{~h}$, with neutrophils being essential for the control of microabscesses and activated macrophages eventually predominating the response before the adaptive immune response enables CD8 T cells to clear the infection (6).

An essential component of this innate immune response is the cytokine response and the elaboration of acute phase proteins by the liver (7). These acute phase proteins are stimulated by cytokines, including interleukin (IL)-1 $\beta$, IL-6, and tumor necrosis factor (TNF).

Serum amyloid A (SAA) proteins are acute phase proteins that have been shown to have specific effects on macrophages and other immune cells. Notably, SAA acts as a chemoattractant for monocytes and neutrophils $(8,9)$, to induce monocyte tissue factor (10), and to induce $\mathrm{T}$ lymphocyte migration and adhesion (11). We hypothesized that a deficiency of acute phase proteins underlies the deficient neonatal murine immune response to listeriosis. In this article, we show that SAA is a critical component of the innate immune response and its deficit contributes to neonatal mortality.

\section{RESULTS}

SAA1, SAA2, and Orosomucoid-2 Are Significantly Higher in Adults Compared With the Levels in Neonates, Both Before and After Listeria Infection

As shown in Figure 1a, a threshold analysis for the five most upregulated genes included SAA1 (Saa1), SAA2 (Saa2), orosomucoid-2 (Orm2), and SAA3 (Saa3). There was a similar increase for Saa 3 in both adults and neonates, but the microarray analysis for the other genes, namely, Saa1, Saa2, and Orm2, suggested that expression was significantly higher at $24 \mathrm{~h}$ in the adult compared with the same in the neonate at all time points.

In Figure 1b, Orm 2 mRNA expression relative to the housekeeping gene Actb ( $\beta$-actin) is significantly increased in the neonate by $48 \mathrm{~h}$, but this expression level is still lower compared with that in the adult mouse at $48 \mathrm{~h}$. The findings for Saal and Saa 2 mRNA expression relative to Actb are more striking, with relative neonatal Saa1 and Saa2 expression significantly less than that in the adult at 24 and $48 \mathrm{~h}$ (Figure 1c,d). Relative expression of Saal and Saa 2 at 24 and $48 \mathrm{~h}$ in the adult is significantly increased compared with that at $0 \mathrm{~h}$ (uninfected adults). 
a
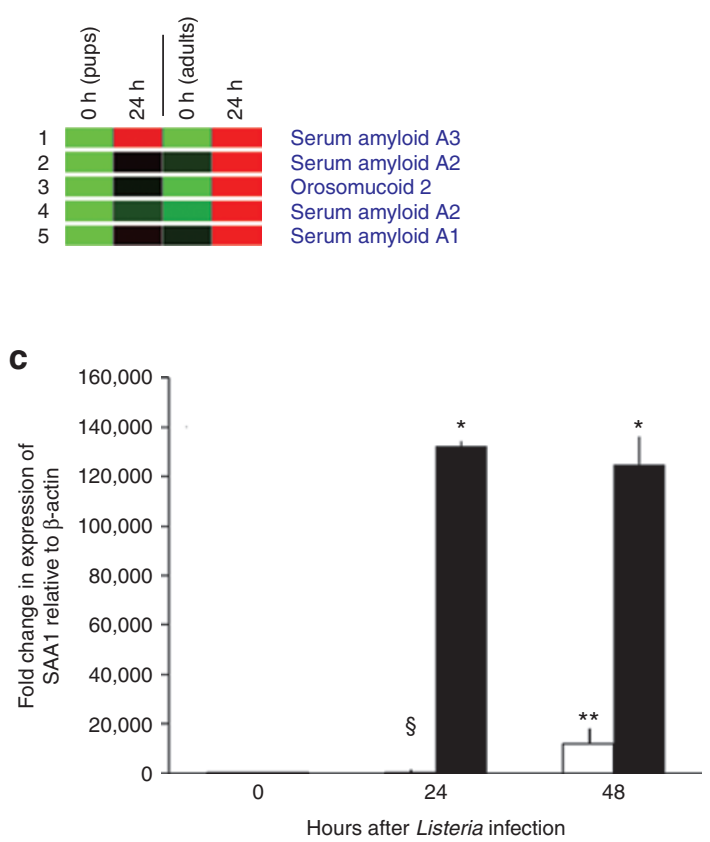

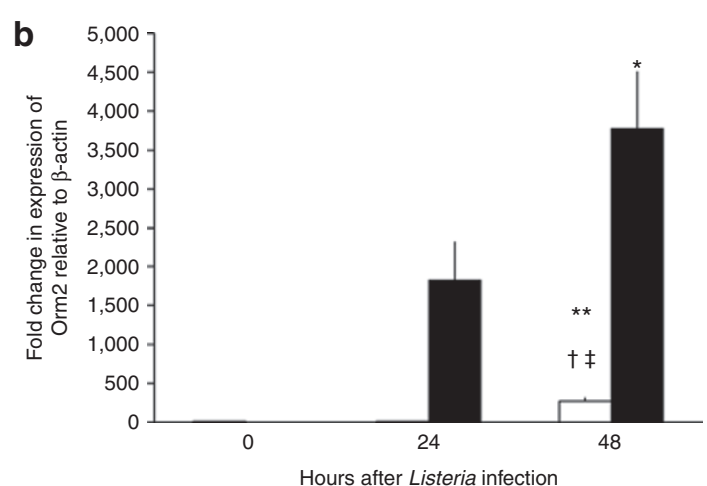

d

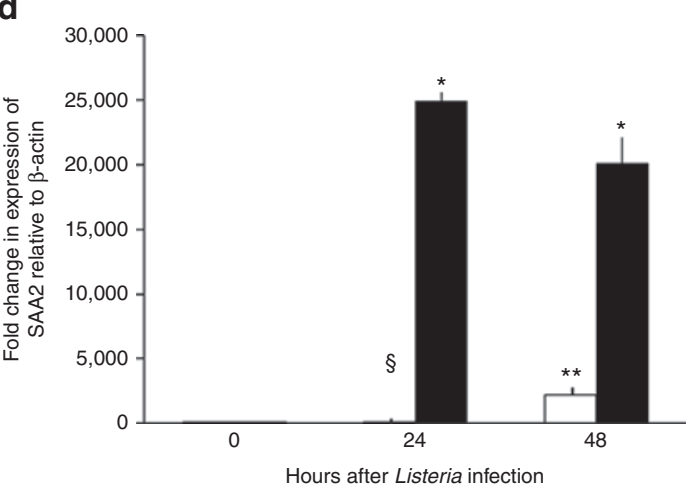

Figure 1. In adult Balb/c mouse liver infected with Listeria monocytogenes, serum amyloid $A$ and orosomucoid-2 are strikingly upregulated relative to uninfected 3- to 5-d-old neonatal mice. To ascertain differences in gene expression between adult and neonatal (3-5 d old) Balb/c mice, RNA was collected from flash-frozen liver before infection $(0 \mathrm{~h})$ and at 24 and $48 \mathrm{~h}$ after infection with an RNeasy Midi Kit (Qiagen, Germantown, MD) and processed on Affymetrix mouse genome 4302.0 microarrays (Affymetrix, Santa Clara, CA). Three adult and neonatal mice were used at each time point. (a) Genesifter web-based software (Geospiza, Seattle, WA) was used for analysis after uploading the raw data with robust multiarray average normalization. The panel represents a threshold analysis showing the five genes with the biggest expression differential between Listeria-infected liver from adults and neonates at 0 and $24 \mathrm{~h}$, as determined using Genesifter software. Note the strong upregulation of the serum amyloid A3, A2, and A1 and orosomucoid-2 proteins in the adult compared with the neonate. Genes whose expression levels are greater are indicated by red, whereas lower expression levels are indicated by light green. (b) The findings from the Affymetrix microarray were confirmed by using real-time reverse-transcription polymerase chain reaction at the uninfected and at the 24 - and 48 -h postinfection time points, with analyses made relative to expression in the uninfected neonate. Expression of orosomucoid-2 (Orm2), as demonstrated using real-time polymerase chain reaction, is upregulated significantly at $48 \mathrm{~h}$ in the adults compared with that in uninfected adults at $0 \mathrm{~h}(*), P$ value $<0.05$. Orm 2 is also upregulated significantly at $48 \mathrm{~h}(* *)$ in the adults compared with that in the neonates, $P$ value $<0.05$. At $48 \mathrm{~h}$, the expression of $\mathrm{Orm} 2$ by the neonates is also upregulated significantly compared with that of the neonates at $0\left(^{+}\right)$ and at $24 \mathrm{~h}\left({ }^{\ddagger}\right), P$ value $<0.05$. Adults are indicated by solid black columns and neonates by white columns; error bars show SEM. (c) Expression of serum amyloid A1 (Saa $)$ is upregulated significantly at $24(P$ value $<0.001)$ and $48 \mathrm{~h}(P$ value $=0.009)$ in the adults compared with that in uninfected adults at $0 \mathrm{~h}\left({ }^{*}\right)$. Saa 1 is also upregulated significantly at $24\left({ }^{5} P\right.$ value $\left.<0.001\right)$ and $48 \mathrm{~h}\left({ }^{* *} P\right.$ value $\left.=0.003\right)$ in the adults compared with the neonates at the same time points. Adults are indicated by solid black columns and neonates by white columns; error bars show SEM. (d) Expression of serum amyloid A2 (Saa2) is upregulated significantly at 24 and $48 \mathrm{~h}$ in the adults compared with that in uninfected adults at $0 \mathrm{~h}(*), P$ values $=0.01$. Saa 2 is also upregulated significantly at $24\left(^{(5)}\right.$ and $48 \mathrm{~h}\left(^{(*)}\right)$ in the adults compared with that same in neonates at the same time points, $P$ values $=0.01$. Adults are indicated by solid black columns and neonates by white columns; error bars show SEM.

\section{TNF Increases in an Exponential Manner in Adults After Listeria Infection, Whereas a Similar Increase Does Not Occur in Neonates}

The course of TNF, IL-6, and IL-1 $\beta$, in response to listeriosis, was analyzed. As shown in Figure 2c, expression of TNF relative to $A c t b$ increases in an exponential manner with time in adults compared with that in the neonates. This contrast is not observed for $I L-6$ and $I L-1 \beta$ relative to Actb (Figure 2a,b).

\section{Prophylactic Intraperitoneal Administration of rhSAA Before Listeriosis Confers a Survival Benefit}

As depicted in Figure 3a, Listeria monocytogenes at a dose of 75 bacteria per gram resulted in the death of all neonates (11 of 11) that had not received recombinant human SAA (rhSAA) within $\sim 3 \mathrm{~d}$, suggesting a deficient innate immune response in the control neonates. However, the prophylactic administration of rhSAA resulted in a clear survival benefit, with 10 of 12 neonates $(83 \%)$ surviving to $168 \mathrm{~h}(7 \mathrm{~d})$. When a dose of 150 Listeria monocytogenes per gram was used (Figure 3b), all neonates not receiving prophylactic rhSAA died within 3-4 d after inoculation, whereas 10 of 16 neonates (62.5\%) ultimately survived. Survival was lower with the use of 200 Listeria monocytogenes per gram (13 vs. $0 \%$; Figure $3 \mathrm{c}$ ), and all mice died within 3-4 d when a dose of at least 250 Listeria monocytogenes per gram was inoculated (Figure 3d).

In all experiments, mice that received only Hanks' balanced salt solution (HBSS) did not survive more than 3-4 d after infection. Survival was assessed at 12 -h intervals, and there was a 24-h window, between 72 and $96 \mathrm{~h}$ after infection, during which the last HBSS-only survivors in each of the four experiments 


\section{Articles $\mid$ Hawkinsetal.}
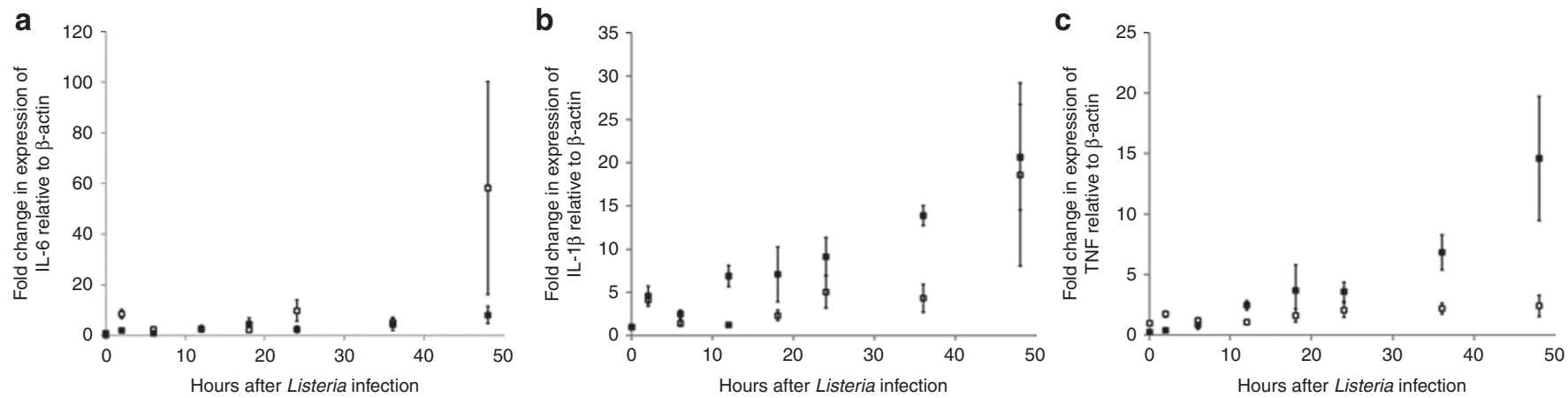

Figure 2. Of the key acute phase cytokines, tumor necrosis factor (TNF) increased in an exponential manner with time in the livers from infected adults, whereas a similar rise is not observed for the neonates. Solid black squares indicate adults, and open squares indicate neonates; error bars show SEM. Liver (four animals at each time point) was flash frozen during collection from uninfected adults and neonates at 2, 6, 12, 18, 24, 36, and 48 h after Listeria monocytogenes infection. RNA was collected using the RNeasy Midi Kit, reverse transcribed, and real-time reverse-transcription polymerase chain reaction was used. Analyses were made relative to expression in the uninfected neonate. $(\mathbf{a}, \mathbf{b})$ There was no differential increase in gene expression for interleukin (IL)- 6 or IL-1 $\beta$ between adults and neonates infected with Listeria. (c) However, gene expression for TNF increased in an exponential manner with time in the infected adult liver, whereas the neonates did not show similar increase.

were noted to have died. The last neonate that received only HBSS and was infected with 75 Listeria per gram died by $72 \mathrm{~h}$ after infection, and less than $20 \%$ of neonates that received only HBSS in the other experiments survived to at least $72 \mathrm{~h}$.

Mice that survived listeriosis after receiving prophylactic rhSAA were more likely to be chronically infected with Listeria monocytogenes. All ten of the neonates that survived for $1 \mathrm{wk}$ after Listeria infection with a dose of 75 Listeria per gram had received prophylactic rhSAA, and all 10 of these mice subsequently survived for $6 \mathrm{wk}$ after initial infection, at which time, they were killed and weighed (mean weight was $14.9 \mathrm{~g}$, with a range of 9.9-18.3 g). Entire spleens were homogenized in $1 \mathrm{ml}$ of sterile phosphate-buffered saline (PBS), and then $100 \mu \mathrm{l}$ of homogenate was plated on tryptic agar plates. The value of $\log _{10}$ of the splenic colony counts was 1.0 for three mice and 2.6 for a single mouse, with the remaining mice showing no evidence of infection. This is similar to the results in nude and thymectomized mice that have increased innate, but no adaptive, immunity (6).

In contrast, only 8 of the 10 neonates that survived listeriosis for at least $1 \mathrm{wk}(168 \mathrm{~h})$ with a dose of 150 Listeria per gram after receiving prophylactic rhSAA survived to $6 \mathrm{wk}$. Importantly, all of these eight survivors showed chronic infection, with $\log _{10}$ splenic colony counts ranging from 1 to 3.5 . These mice were significantly smaller at $6 \mathrm{wk}$ of age compared with the mice that had received a dose of 75 Listeria per gram $(P=0.001)$, with a mean weight of $11.4 \mathrm{~g}$ (range: 9.8-13.4 $\mathrm{g}$ ).

\section{rhSAA Did Not Affect Postnatal Growth, as Determined by Body Weight at 5 Wk of Life}

As described earlier, mice that had received rhSAA and survived listeriosis showed postnatal growth restriction, and therefore we sought to exclude rhSAA being a cause of the postnatal growth restriction. As shown in Figure 4a, the mean weight of uninfected mice that had received rhSAA in HBSS was $9.0 \mathrm{~g}(n=10)$, whereas the mean weight of uninfected mice that received only HBSS was $7.9 \mathrm{~g}(n=11)$; this difference was not significant $(P=0.3)$.

\section{Mice That Received Prophylactic rhSAA Had Lower Listeria Colony Counts in the Liver After Infection}

As presented in Figure $4 \mathrm{~b}$, mice that received prophylactic rhSAA had lower Listeria colony counts compared with mice that did not receive rhSAA. Specifically, at $24 \mathrm{~h}$, mice that received prophylactic rhSAA had $86 \%$ lower colony counts: logarithmic mean $=4.3$ (range: $4.1-4.6)$ vs. 5.2 (range: 4.9 5.3), $P=0.004$; and at $48 \mathrm{~h}$, mice that received prophylactic rhSAA had $98 \%$ lower colony counts: logarithmic mean $=5.6$ (range: $5.5-5.6$ ) vs. 7.3 (range: 7.2-7.5), $P<0.001$. Mice that did not receive rhSAA were more likely to appear jaundiced and lethargic in appearance at the time of killing.

\section{Greater Numbers of Activated Macrophages Are Present in the} Livers of Neonatal Mice That Received Prophylactic rhSAA Before Listeria Infection

Mice that received prophylactic rhSAA had greater average numbers of activated macrophages per high-power field (HPF; magnification of $\times 40$ ) compared with mice that did not receive prophylactic rhSAA (10 HPFs analyzed per mouse). As seen in Figure 4c, in uninfected liver (0h, which is $18 \mathrm{~h}$ after intraperitoneal (i.p.) injection with either rhSAA in HBSS or HBSS alone), mice $(n=1)$ that received rhSAA had 5.4 activated macrophages per HPF (range: $1-10$ ) vs. 2.5 activated macrophages per HPF (range: $0-7)$ in mice $(n=3)$ that did not receive prophylactic rhSAA $(P=0.009)$. Similarly, at $12 \mathrm{~h}$ after Listeria infection, mice $(n=4)$ that had received prophylactic rhSAA had a mean of 9.9 activated macrophages per HPF (range: 5-22) compared with 3.3 activated macrophages per HPF (range: 0-6) in mice that did not receive prophylactic rhSAA $(n=4)$, and this difference was statistically significant $(P<0.001)$. At $24 \mathrm{~h}$ after Listeria infection, mice that had received prophylactic rhSAA $(n=4)$ had a greater number of activated macrophages per HPF (mean: 7.5; range: 1-20) compared with mice $(n=4)$ that did not receive prophylactic rhSAA (mean: 4.9; range: 1-17), and this was also statistically significant $(P=0.007)$. At $48 \mathrm{~h}$ after Listeria infection, the counts of activated macrophages were no longer statistically 
a

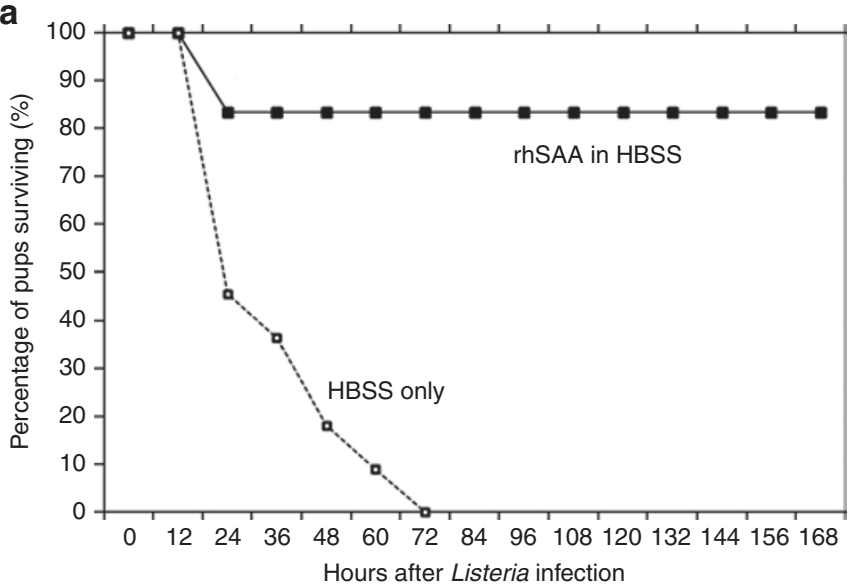

C

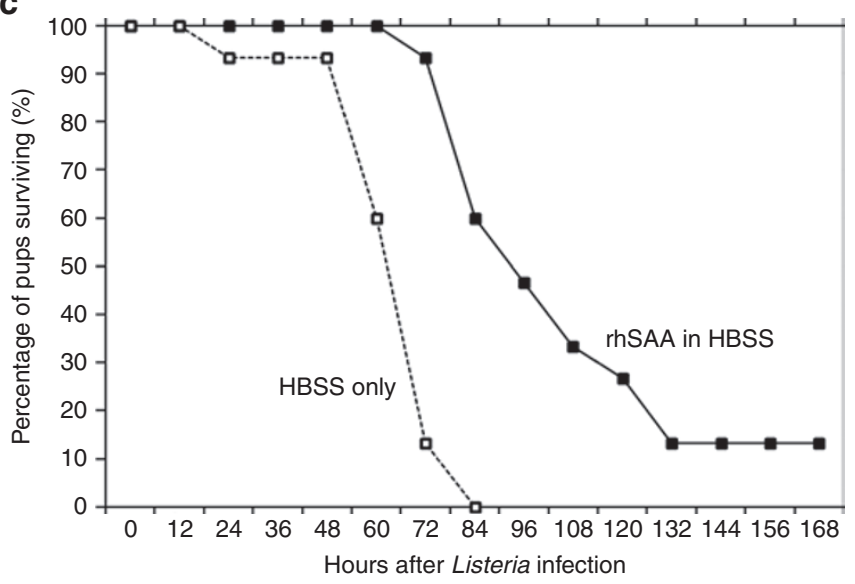

b

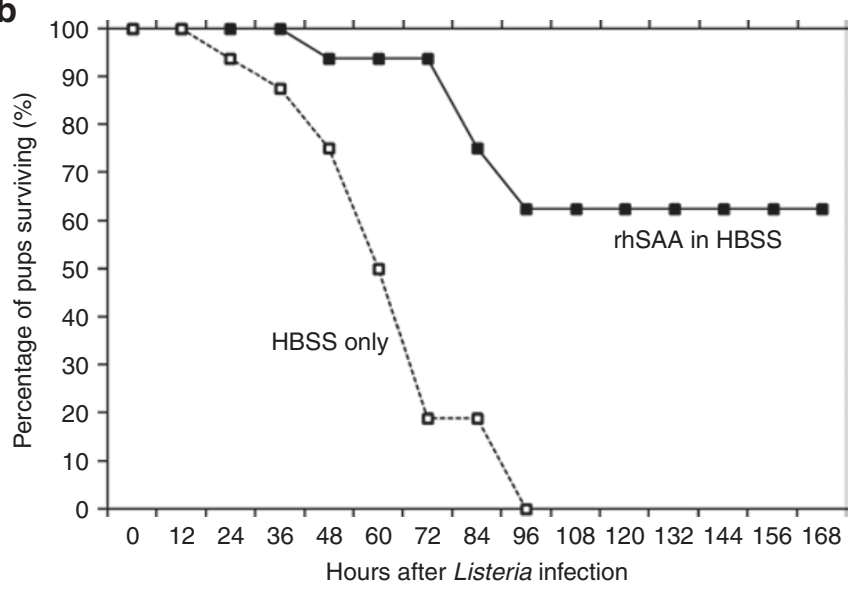

d

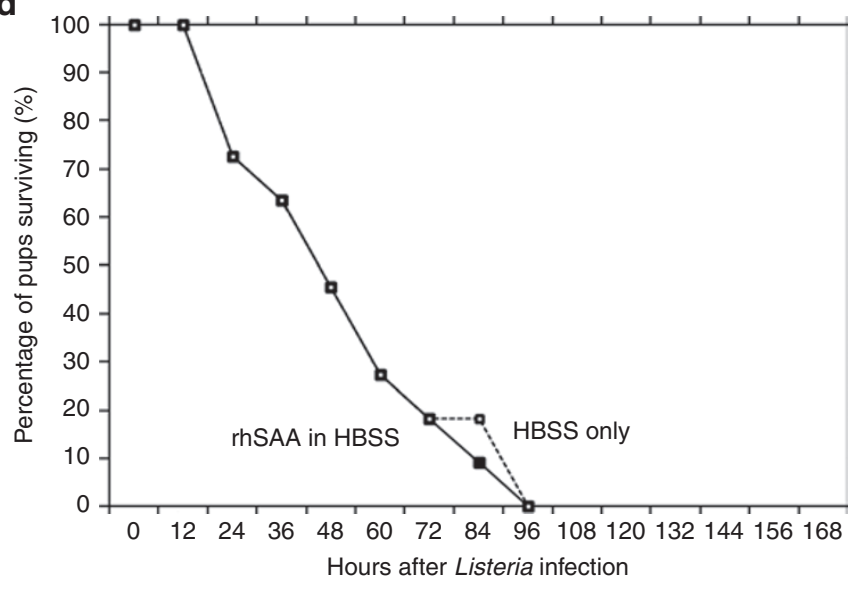

Figure 3. Neonatal Balb/c mice (3-5 d old) that received recombinant human serum amyloid A ( $\mathrm{hSAA}$ ) intraperitoneally (i.p.) $18 \mathrm{~h}$ before infection with various doses of Listeria monocytogenes showed a dramatic survival benefit compared with age- and weight-balanced infected controls that received only vehicle (Hanks' balanced salt solution (HBSS)) before infection. Eighteen hours before infection, two groups of 3- to 5-d-old pups balanced by weight were injected (i.p.) with $50 \mu \mathrm{l}$ of either recombinant human serum amyloid A (rhSAA) in HBSS $(50 \mu \mathrm{g} / \mathrm{ml})$ or only HBSS. At the time of infection, doses of 75 Listeria per gram, 150 Listeria per gram, 200 Listeria per gram, and at least 250 Listeria per gram were injected i.p. into the pups. Survival was followed for $168 \mathrm{~h}$ ( $1 \mathrm{wk}$ ) after infection. (a) Among the controls that received only vehicle, listeriosis was uniformly fatal within 3-4 d after infection ( $n=11$ ). In contrast, after infection with a dose of 75 Listeria per gram, $83 \%$ of neonates that received rhSAA $(n=12)$ survived at least 1 wk. (b) After a dose of 150 Listeria per gram, $62.5 \%$ of neonates that received rhSAA $(n=16)$ survived at least $1 \mathrm{wk}$, whereas the controls that received only vehicle $(n=16)$ died within 3-4 d after infection. (c) After a dose of 200 Listeria per gram, $13 \%$ of neonates survived at least 1 wk $(n=15)$, whereas the controls that received only vehicle $(n=15)$ died within 3-4 d after infection. (d) Above a dose of 250 Listeria per gram, prophylactic rhSAA was not protective, and all mice died within 3-4 d of infection ( $n=11$ in both groups).

significant. Supplementary Figure S1 online depicts representative images that show increased macrophage activation for neonates that received prophylactic rhSAA at the time of infection (18 h after receiving rhSAA, Supplementary Figure S1a) and at $12 \mathrm{~h}$ after infection (Supplementary Figure S1b), and $24 \mathrm{~h}$ after infection (Supplementary Figure S1c), whereas Supplementary Figure S1d-f show corresponding representative images for mice that did not receive prophylactic rhSAA (only vehicle). Supplementary Figure S1g-i shows representative negative control slides.

\section{DISCUSSION}

Although listeriosis is not a common cause of infection within the general population, it is a problem for specific subgroups, in particular neonates, pregnant women, and elderly adults (12). Similar to humans, neonatal mice are at increased risk for listeriosis compared with adults, and thus our model of listeriosis is a relevant and useful one. In the case of neonatal mice, this increased susceptibility to listeriosis has been associated with a defect in the innate immune response, specifically a deficiency of activated macrophages bearing MHC class-II (Ia) (3), and unpublished data from our laboratory indicate that the neutrophilic response to acute listeriosis is similar in neonates and adults. We found that gene expression for SAA proteins was deficient in the neonate compared with that in the adult mouse after infection with Listeria monocytogenes. SAA has important immune effector functions and has been shown to function as a chemoattractant for monocytes and neutrophils $(8,9)$, to induce monocyte tissue factor $(10)$, and to induce $\mathrm{T}$ lymphocyte migration and adhesion (11). Thus, the presence of the SAA proteins in listeriosis is likely an important factor in the timeline, as described by Unanue (6), whereby 

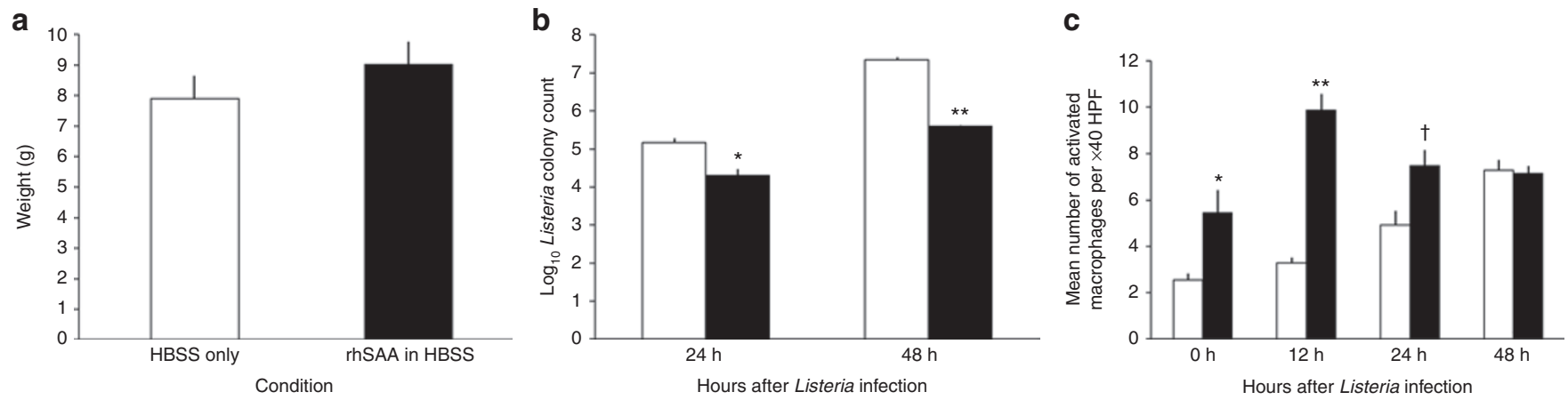

Figure 4. Recombinant human serum amyloid A (rhSAA) did not affect postnatal growth but did result in fewer Listeria colony counts, as measured by plating infected liver, and in greater numbers of activated macrophages in uninfected and infected neonatal liver. Error bars show SEM. (a) Neonates were divided into two groups balanced by age and body weight, then injected with either $50 \mu \mathrm{l} \mathrm{of} \mathrm{rhSAA} \mathrm{(} 50 \mu \mathrm{g} / \mathrm{ml}$ in Hanks' balanced salt solution (HBSS)) or $50 \mu \mathrm{l}$ of HBSS alone. These mice were then killed after $5 \mathrm{wk}$, and their body weights were compared. There was no significant difference in postnatal growth as measured by body weight at 5 postnatal weeks $(9.0 \mathrm{~g}$ for mice that received rhSAA in HBSS, $n=10$; vs. $7.9 \mathrm{~g}$ for mice that received only HBSS, $n=11 ; P$ value $=0.3$ ). (b) Neonatal Balb/c mice ( $4 \mathrm{~d}$ old) were divided into two groups, with 3-4 mice in each group for each time point and were balanced by body weight. The mice received intraperitoneal (i.p.) injection of either rhSAA ( $50 \mathrm{mg} / \mathrm{ml})$ in HBSS or HBSS alone $(50 \mu$ l total volume). After $18 \mathrm{~h}$, the mice received i.p. injection of 150 Listeria per gram and were killed at 24 and $48 \mathrm{~h}$ after infection. Livers were homogenized with sterile phosphate-buffered saline and plated on tryptic soy agar plates. Neonates that received rhSAA, compared with controls that received only vehicle, showed significantly fewer $\log _{10}$ Listeria colony counts after plating on tryptic soy agar plates (log 5.2 vs. 4.3 at 24 h post-Listeria infection, ${ }^{*} P=0.004 ; 7.3$ vs. 5.6, ${ }^{* *} P<0.001$ at $48 \mathrm{~h}$ post-Listeria infection). (c) Neonatal Balb/c mice (3-5 d old) were divided into two groups balanced by weight and received i.p. injection of either rhSAA $(50 \mathrm{mg} / \mathrm{ml})$ in HBSS or HBSS alone $(50 \mu \mathrm{l}$ total volume). After $18 \mathrm{~h}$, the mice received i.p. injection of 150 Listeria per gram and were killed at 0,12 , 24 , and $48 \mathrm{~h}$ after infection. Livers were preserved using periodate-lysine-paraformaldehyde, serially sectioned, and stained for major histocompatibility complex (MHC) class-Il antibody as a marker of macrophage activation. Ten high-power fields (HPFs) were counted per neonate. Compared with mice $(n=3)$ that received only HBSS, a neonate that received rhSAA $(n=1) 18 \mathrm{~h}$ before killing showed significantly greater numbers of activated macrophages per HPF, as determined by signal MHC class-II staining, in uninfected liver at $0 \mathrm{~h},{ }^{*} P=0.009$. There were also significantly greater numbers of activated macrophages per HPF at 12 and $24 \mathrm{~h}$ postinfection in mice that received rhSAA in HBSS $18 \mathrm{~h}$ before infection $(n=4$ mice per group) compared with mice that received only HBSS ( $n=4$ mice per group), ${ }^{* *} P<0.001(12 \mathrm{~h})$ and ${ }^{\dagger} P=0.007(24 \mathrm{~h})$. There was no significant difference in the number of activated macrophages per HPF at $48 \mathrm{~h}$ postinfection in mice that received rhSAA in HBSS $18 \mathrm{~h}$ before infection $(n=8$ mice) compared with mice that received only HBSS ( $n=4$ mice), $P=0.81$.

the initial arrival of neutrophils is complemented by activated macrophages, with eventual transition to the adaptive immune response enabling CD8 T cells to clear the infection.

We found that expression of hepatic TNF increased in an exponential manner with time after Listeria infection of adults, but a similar rise was not found in infected neonates. Previously, it has been reported that (i) newborn rats infected with Listeria monocytogenes had deficient TNF levels, (ii) pretreatment of juvenile, but not newborn rats, with exogenous TNF decreased the splenic bacterial load, (13) and (iii) TNF is important for control of murine listeriosis (14). Importantly, neonatal mice that are deficient in the TNF receptor-1 show large necrotic lesions of neutrophils and macrophages in the liver and are unable to switch to a T-cell-mediated sterilizing response (15), suggesting a crucial role for TNF in the neonatal response to listeriosis. The strain of Listeria monocytogenes can influence regulation of TNF production, such that a less pathogenic, non-listeriolysin (LLO-)-producing strain results in sustained transcription of TNF (16). In this study, the Listeria strain that was used was a listeriolysin-producing (LLO+) strain.

Acute phase cytokines other than TNF also influence the innate immune response. IL-6-deficient mice have a defect in the neutrophilic response $(17,18)$, but this deficiency does not affect macrophage activation (17). Consistent with this, we did not see a differential increase in $I L-6$ expression between adults and neonates, and there was no apparent difference in the neutrophilic response to Listeria-infected liver and spleen. Nor did we find a differential rise in $I L-1 \beta$ expression in adult mice compared with the expression in neonatal mice. Importantly, IL-1 $\beta$ influences macrophage activation and, thus, the innate immune response (19). We can thus conclude that the neonatal deficiency in macrophage activation is not a result of differences in IL-1 $\beta$ but is likely related to deficient neonatal production of TNF, which in turn may underlie deficient SAA expression because we show that prophylactic recombinant SAA increases macrophage activation.

In addition to the gene expression for TNF and the SAA proteins, we found the expression of orosomucoid-2 (Orm2), an $\alpha-1$-acid glycoprotein and acute phase protein, to be deficient in the neonate. Hepatic expression of Orm2, Saa1, and Saa2 increases in response to Plasmodium chabaudi malaria infection (20). Other factors have been reported to affect the susceptibility of neonatal mice to infection. In particular, docosahexaenoic acid, found in rodent fetal serum and diets rich in fish oil, inhibits acquisition of macrophage activation and cyclooxygenase-based tumoricidal function (21). Interestingly, we found that mice that received rhSAA and that subsequently survived listeriosis were more likely to be chronically infected. This is similar to the situation of nude and thymectomized mice, which survive acute listeriosis but remain chronically infected. Due to a deficiency of CD8 $\mathrm{T}$ lymphocytes, nude mice are unable to sterilize the bacteria (6). An understanding of why neonates surviving 
with rhSAA were chronically infected with Listeria will be important.

We report that SAA is a critical factor in the acute phase innate immune response. Neonatal mice infected with Listeria monocytogenes showed deficient gene expression for SAA 1 and 2 compared with infected adults, and this was correlated with deficient gene expression of TNF, deficient macrophage activation, and higher Listeria burdens. Although our findings do not prove that the increased susceptibility of neonatal mice is entirely due to lack of SAA upregulation, it may be that ultimately SAA could be used as a therapeutic adjunct in the case of neonatal sepsis. We report that when neonatal mice were pretreated with recombinant SAA, there was a considerable survival benefit associated with a decreased bacterial load and greater numbers of activated macrophages.

\section{METHODS}

\section{Balb/c Mice}

$\mathrm{Balb} / \mathrm{c}$ adult mice (at least $6 \mathrm{wk}$ old) and their pups were bred and maintained under pathogen-free conditions in our vivarium in accordance with the Institutional Animal Care and Use Committee of the University of Texas Southwestern Medical Center. Adult mice were killed by the protocol using $\mathrm{CO}_{2}$ narcotization with cervical dislocation, and neonatal mice were killed by decapitation.

\section{Listeriosis Model}

The methods for culturing and counting the Listeria monocytogenes (strain CNL 85/163) were described previously (16). The Listeria strain was injected i.p. using a 1-ml U-100 insulin syringe with a 30-gauge needle. Doses of Listeria monocytogenes used were based on the unpublished work by scientists at our laboratory showing that similar bacterial colony counts were obtained with $4.2 \times 10^{5}$ total Listeria per adult mouse and 150 Listeria per gram for 3- to 5-d-old neonatal mice (greater than 1,000-fold difference for the example of a 2.5-g neonate), emphasizing the dramatically increased susceptibility of neonatal mice to infection. In neonatal mice, great care was taken to avoid deep i.p. injection toward the viscera or across the central abdominal vessels.

\section{Histology and Immunohistopathology}

Liver and spleen were fixed using periodate-lysine-paraformaldehyde and frozen in Tissue-Tek O.C.T. Compound (Sakura Finetek, Torrance, CA). Serial frozen sections of $8-\mu \mathrm{m}$ thickness were cut using a cryostat-microtome. Slides were dipped in $70 \%$ ethanol for $1 \mathrm{~min}$ and then rinsed by dipping in 1X PBS 10 times. A blocking solution containing 3.3\% normal goat serum (Vector Labs, Burlingame, CA), 3.3\% normal donkey serum (Jackson Immunoresearch, West Grove, PA), and 3.3\% bovine serum albumin (Sigma-Aldrich, St Louis, MO) in 1X PBS was applied for $1 \mathrm{~h}$, and the slides were incubated with the primary antibody overnight at $4{ }^{\circ} \mathrm{C}$, washed three times with $1 \mathrm{X}$ PBS, and incubated with the secondary antibody for $1 \mathrm{~h}$ at room temperature in the dark. For MHC class-II and Listeria staining, a 1:100 dilution of rat anti-mouse MHC class-II IgG2b primary antibody (Clone ER-TR3; BMA Biomedicals, Augst, Switzerland) and a 1:50 dilution of rabbit anti-Listeria IgG primary antibody (ViroStat, Portland, ME), respectively, were applied. Negative control slides used only secondary antibodies without primary antibodies. Secondary antibodies included AlexaFluor-488 goat anti-rat IgG (Invitrogen, Carlsbad, CA) and Cy3-conjugated goat anti-rabbit IgG (Jackson Immunoresearch, West Grove, PA). After staining with secondary antibodies, the slides were dipped in Milli-Q water 10 times, and a cover slip was applied using Vectashield with DAPI (Vector Labs, Burlingame, CA); the slides were visualized with a Zeiss-LSM510 microscope.

\section{Affymetrix Quantitative Real-Time Reverse-Transcription PCR}

Liver was removed after killing of the animal and was immediately flash frozen in liquid nitrogen and stored at $-80^{\circ} \mathrm{C}$. RNA was extracted from murine liver using the RNeasy Midi Kit (Qiagen, Germantown, MD) according to the manufacturer's protocol. Residual DNA was removed by treatment with Turbo DNase (ABI, Foster City, CA). First-strand cDNAs were synthesized from equal amounts of total RNA $(2 \mu \mathrm{g})$ using the Applied Biosystems high-capacity cDNA Reverse Transcription Kit (ABI, Foster City, CA). For Affymetrix gene chip analysis, RNA was delivered to our university's Microarray Core Facility for processing on Mouse Genome 430 2.0-Array chips (Affymetrix, Santa Clara, CA), and the raw data were then analyzed after uploading to the Genesifter website (Geospiza, Seattle, WA) using robust multiarray average normalization. Results were confirmed by analyzing for the expression of SAA1 (Saa1), SAA2 (Saa2), and orosomucoid-2 (Orm2) transcripts, relative to $\beta$-actin $(A c t b)$, by real-time reverse-transcription PCR, using appropriate primers (Integrated DNT Technologies, Coralville, IA). Transcripts for the acute phase cytokines, including IL-1 $\beta$ (Il1b), Tnf, and IL-6 (Il6), relative to $\beta$-actin $(A c t b)$, were also analyzed by real-time reverse-transcription PCR. Primers included the following for the genes indicated: Saa1 (Sense: 5'-TGC-CTG-ACA-AAT-ACT-GAG-CGT-CCT-3', Antisense: 5'-CCC-TTG-TGG-GAT-ACA-CAC-ATG-AGA-GA-3'), Saa2 (Sense: 5'-TGG-ACT-GCC-TGC-CAA-ATA-CTG-A-3', Antisense: 5'-GTGGGA-TAC-ACA-CAT-GAG-AGG-T-3'), Orm2 (Sense: 5'-ATC-TCTTCC-AAG-CCC-TGG-TG-3', Antisense: 5'-ATT-GGT-GAT-AGGGTC-GCC-TAT-GGT-3'), Il1b (Sense: 5'-AAG-GGC-TGC-TTCCAA-ACC-TTT-GAC-3', Antisense: 5'-ATA-CTG-CCT-GCC-TGAAGC-TCT-TGT-3'), Tnf (Sense: 5'-TCT-CAT-GCA-CCA-CCA-TCAAGG-ACT-3', Antisense: 5'-ACC-ACT-CTC-CCT-TTG-CAG-AACTCA-3'), Il6 (Sense: 5'-GGA-CCA-AGA-CCA-TCC-AAT-TCA-3', Antisense: 5'-CGC-ACT-AGG-TTT-GCC-GAG-TAG-3'), and Actb (Sense: 5'-GGC-TGT-ATT-CCC-CTC-CAT-CG-3', Antisense: 5'-CCA-GTT-GGT-AAC-AAT-GCC-ATG-T-3'). Amplification was performed using an ABI-Prism 7000 Sequence Detector (ABI), with one cycle of $95^{\circ} \mathrm{C}$ for $3 \mathrm{~min}$, followed by 40 cycles of $95^{\circ} \mathrm{C}$ for $15 \mathrm{~s}$ and $60^{\circ} \mathrm{C}$ for $45 \mathrm{~s}$. The reaction was performed in triplicate for each sample using iTaq SYBR Green Supermix with Rox (Bio-Rad, Hercules, CA). The $\Delta \Delta \mathrm{Ct}$ method was used for data analysis. Expression was averaged for at least three adults and three neonates for each time period, and expression was analyzed relative to that of the uninfected neonate. Statistical comparisons were made using two-tailed Student's $t$-tests with unequal variance.

\section{Survival Analyses and Effects of rhSAA}

Recombinant human apo-SAA (rhSAA) was obtained from PeproTech (Rocky Hill, NJ) and reconstituted in water to a concentration of $1.0 \mathrm{mg} / \mathrm{ml}$. The rhSAA was diluted in HBSS (Invitrogen, Grand Island, $\mathrm{NY}$ ) to a concentration of $50 \mu \mathrm{g} / \mathrm{ml}$, and a $50-\mu \mathrm{l}$ volume was administered i.p. into the treated 3- to 5-d-old neonatal mice, whereas a 50- $\mu \mathrm{l}$ aliquot of HBSS was given i.p. into weight- and age-balanced controls. Eighteen hours after these i.p. injections, the mice received a dose of $75,150,200$, or at least 250 Listeria per gram i.p. Survival was followed for $1 \mathrm{wk}(168 \mathrm{~h})$ at 12 -h intervals after listeriosis infection. The concentration of $50 \mu \mathrm{g} / \mathrm{ml}$ was selected based on work by previous investigators $(8,9,11)$. To assess the effect of rhSAA on postnatal growth, 3- to 5-d-old neonates were injected i.p. either with $50 \mu \mathrm{l}$ of rhSAA diluted in HBSS (Invitrogen) to a concentration of $50 \mu \mathrm{g} / \mathrm{ml}$ or with $50 \mu \mathrm{l}$ of HBSS into weight- and age-balanced controls. These mice did not receive Listeria but were housed with the dam, killed at $5 \mathrm{wk}$ of age, and weight comparisons were made. To assess differences in Listeria counts, neonatal Balb/c mice ( $4 \mathrm{~d}$ old) balanced by body weight were divided into groups, with 3-4 mice per intervention at each time point. These mice received either rhSAA in HBSS or only HBSS at $18 \mathrm{~h}$ before i.p. inoculation with 150 Listeria per gram and then were killed 24 and $48 \mathrm{~h}$ later. Livers were homogenized in sterile PBS and plated on tryptic soy agar plates for colony counting. To assess the effect of rhSAA on MHC class-II expression in the murine liver, neonates received either rhSAA in HBSS or only HBSS at $18 \mathrm{~h}$ before i.p. inoculation with 150 Listeria per gram and were then killed 0, 12, 24, and $48 \mathrm{~h}$ later; the liver was fixed with periodate-lysine-paraformaldehyde and processed and stained for MHC class-II expression as described earlier. The average numbers of activated macrophages per high-power field (magnification of $\times 40$ ), with 10 high-power fields counted per mouse per time point, were compared in an unblinded manner. 


\section{Articles $\mid$ Hawkins et al.}

\section{Statistics}

Statistical comparisons were made using two-tailed Student's $t$-tests with unequal variance. A $P$ value of 0.05 or less was considered significant.

\section{SUPPLEMENTARY MATERIAL}

Supplementary material is linked to the online version of the paper at http://www.nature.com/pr

\section{ACKNOWLEDGMENTS}

We thank Jianlin Chen for her thoughtful suggestions.

\section{STATEMENT OF FINANCIAL SUPPORT}

Supported by National Institutes of Health grant 1R01DK069633 to Christopher Lu.

\section{REFERENCES}

1. Kenzel S, Henneke P. The innate immune system and its relevance to neonatal sepsis. Curr Opin Infect Dis 2006;19:264-70.

2. Levy O. Innate immunity of the newborn: basic mechanisms and clinical correlates. Nat Rev Immunol 2007;7:379-90.

3. Lu CY, Calamai EG, Unanue ER. A defect in the antigen-presenting function of macrophages from neonatal mice. Nature 1979;282:327-9.

4. Lu CY, Peters E, Unanue ER. Ia-bearing macrophages in athymic mice antigen presentation and regulation. J Immunol 1981;126:2496-8.

5. Seo SK, Jeong HY, Park SG, et al. Blockage of endogenous B7-H1 suppresses antibacterial protection after primary Listeria monocytogenes infection. Immunol 2007;123:90-9.

6. Unanue ER. Studies in listeriosis show the strong symbiosis between the innate cellular system and the T-cell response. Immunol Rev 1997;158: $11-25$.

7. Suffredini AF, Fantuzzi G, Badolato R, Oppenheim JJ, O’Grady NP. New insights into the biology of the acute phase response. J Clin Immunol 1999;19:203-14.

8. Badolato R, Johnston JA, Wang JM, et al. Serum amyloid A induces calcium mobilization and chemotaxis of human monocytes by activating a pertussis toxin-sensitive signaling pathway. J Immunol 1995;155: 4004-10.
9. Badolato R, Wong JM, Murphy WJ, et al. Serum amyloid-A is a chemoattractant: induction of migration, adhesion, tissue infiltration of monocytes and polymorphonuclear leukocytes. J Exper Med 1994;180:203-9.

10. Cai H, Song C, Endoh I, et al. Serum amyloid A induces monocyte tissue factor. J Immunol 2007;178:1852-60.

11. Xu L, Badolato R, Murphy WJ, et al. A novel biologic function of serum amyloid A. Induction of T lymphocyte migration and adhesion. J Immunol 1995;155:1184-90.

12. Swaminathan B, Gerner-Smidt P. The epidemiology of human listeriosis. Microbes Infect 2007;9:1236-43.

13. Bortolussi R, Rajaraman K, Serushago B. Role of tumor necrosis factoralpha and interferon-gamma in newborn host defense against Listeria monocytogenes infection. Pediatr Res 1992;32:460-4.

14. Virna S, Deckert M, Lütjen S, et al. TNF is important for pathogen control and limits brain damage in murine cerebral listeriosis. J Immunol 2006;177:3972-82.

15. Sonje MB, Abram M, Stenzel W, Deckert M. Listeria monocytogenes (delta-actA mutant) infection in tumor necrosis factor receptor p55-deficient neonatal mice. Microb Pathog 2010;49:186-95.

16. Vazquez MA, Sicher SC, Wright WJ, et al. Differential regulation of TNFalpha production by listeriolysin-producing versus nonproducing strains of Listeria monocytogenes. J Leuk Biol 1995;58:556-62.

17. Dalrymple SA, Lucian LA, Slattery R, et al. Interleukin-6-deficient mice are highly susceptible to Listeria monocytogenes infection: correlation with inefficient neutrophilia. Infect Immun 1995;63:2262-8.

18. Kopf M, Baumann H, Freer G, et al. Impaired immune and acute-phase responses in interleukin-6-deficient mice. Nature 1994;368:339-42.

19. Rogers HW, Sheehan KC, Brunt LM, Dower SK, Unanue ER, Schreiber RD. Interleukin 1 participates in the development of anti-Listeria responses in normal and SCID mice. Proc Natl Acad Sci USA 1992;89:1011-5.

20. Delic D, Ellinger-Ziegelbauer H, Vohr HW, Dkhil M, Al-Quraishy S, Wunderlich F. Testosterone response of hepatic gene expression in female mice having acquired testosterone-unresponsive immunity to Plasmodium chabaudi malaria. Steroids 2011;76:1204-12.

21. Dustin LB, Shea CM, Soberman RJ, Lu CY. Docosahexaenoic acid, a constituent of rodent fetal serum and fish oil diets, inhibits acquisition of macrophage tumoricidal function. J Immunol 1990;144:4888-97. 